\title{
Precious Metal-Free Photocatalytic Water Oxidation by a Layered Double Hydroxide-Prussian Blue Analogue Hybrid Assembly
}

\author{
Sina Sadigh Akbari ${ }^{[a]}$ and Ferdi Karadas ${ }^{*[a, b]}$
}

The development of earth-abundant photocatalytic assemblies has been one of the bottlenecks for the advancement of scalable water splitting cells. In this study, a $\mathrm{ZnCr}$ layered double hydroxide and a CoFe Prussian blue analogue are combined to afford an earth-abundant photocatalytic assembly involving a visible light-absorbing semiconductor (SC) and a water oxidation catalyst (WOC). Compared to bare $\mathrm{ZnCr}-\mathrm{LDH}$, the SC-WOC hybrid assembly exhibits a threefold enhancement in photocatalytic activity, which is maintained for $6 \mathrm{~h}$ under photocatalytic conditions at $\mathrm{pH}$. The band energy diagram was extracted from optical and electrochemical studies to elucidate the origin of the enhanced photocatalytic performance. This study marks a straightforward pathway to develop low-cost and precious metal-free assemblies for visible lightdriven water oxidation.

\section{Introduction}

Photocatalytic water oxidation with powder suspensions has been an appealing strategy since it offers a simple, relatively low-cost, and easily scalable method to harvest solar light. These photocatalytic assemblies generally involve the coupling of a suitable semiconductor (SC) with a water oxidation catalyst (WOC). ${ }^{[1,2]}$ Although hundreds of different assemblies have been reported up to date, the majority of them are active mostly in the UV region $(<400 \mathrm{~nm})$, and/or WOCs are mainly based on precious metal oxides. ${ }^{[3-5]}$ Therefore, the development of an earth-abundant and visible-light absorbing SC-WOC assembly is one of the critical challenges for the advancement of scalable and efficient photocatalytic water splitting systems.

Layered double hydroxides (LDHs) consisting of mixedmetal hydroxide layers step forward in photocatalytic water splitting due to their simple synthesis, diversity, abundant active earth-metal centers, and efficient visible light-harvesting properties. ${ }^{[6-13]}$ Although LDHs have been actively used for photocatalytic water splitting due to their high quantum yield and efficiency, only a limited number of studies involve the utilization of $\mathrm{ZnCr}$-LDH as a narrow bandgap semiconductor for the photocatalytic water oxidation process. ${ }^{[14]} \mathrm{ZnCr}-\mathrm{LDH}$ has been employed either as an individual photocatalyst ${ }^{[15-17]}$ or as part of a heterostructure consisting of a combination of suitable semiconductors for overall water splitting. ${ }^{[18-20]}$ Although $\mathrm{ZnCr}$ -

[a] S. S. Akbari, Prof. F. Karadas

Department of Chemistry, Faculty of Science

Bilkent University, 06800, Ankara (Turkey)

E-mail: karadas@fen.bilkent.edu.tr

[b] Prof. F. Karadas

UNAM - National Nanotechnology Research Center, Institute of Materials Science and Nanotechnology

Bilkent University, 06800, Ankara (Turkey)

Supporting information for this article is available on the WWW under https://doi.org/10.1002/cssc.202002279
LDH exhibits a suitable band energy alignment for visible lightdriven water oxidation, poor charge mobility in LDHs leads to unavoidable recombination of photo-induced charge carriers, which yields relatively poor efficiency and stability. ${ }^{[14]}$ Therefore, viable strategies to suppress photo-generated electron-hole recombination and to improve the interfacial kinetics of $\mathrm{LDH}$ based materials should be introduced for the advancement of LDH-based water splitting technologies. ${ }^{[21-24]}$ Although $\mathrm{ZnCr}$ LDH has been coupled with other semiconductors for photocatalytic applications, it has not been combined with a water oxidation catalyst up to date.

Such a design requires the coupling of two earth-abundant components with matching band levels for efficient charge separation. Cobalt-iron Prussian blue analogues, CoFe-PBAs, have recently been acknowledged as efficient and robust WOCs. In CoFe-PBAs, cobalt sites, which are coordinated to at least one water molecule, are active catalytic sites while iron sites that are surrounded with six cyanide groups are catalytically inactive. ${ }^{[25-31]}$ Since they exhibit poor photocurrent efficiencies, the general strategy is to couple CoFe-PBAs with suitable photosensitizers and semiconductors to utilize them for the light-driven water oxidation process..$^{[27,32]}$ Herein, we prepare SCWOC hybrid assembly by coupling a CoFe-PBA with a $\mathrm{ZnCr}-\mathrm{LDH}$ since i) they are precious metal free, ii) they can be prepared by straightforward solution chemistry, and iii) CoFe-PBAs are projected to have a suitable band energy alignment that can facilitate charge transfer from the valence band of $\mathrm{ZnCr}-\mathrm{LDH}$ for the water oxidation reaction. ${ }^{[33-36]}$

\section{Results and Discussion}

$\mathrm{ZnCr}$-LDHs and CoFe-PBAs have been prepared according to previously reported synthetic methods. The compounds were then mixed at $60^{\circ} \mathrm{C}$ with different weight ratios to afford hybrid assemblies symbolized as LDH-PB- $n$ ( $n=1,2$, or 3; Figure 1a). All 
(a)

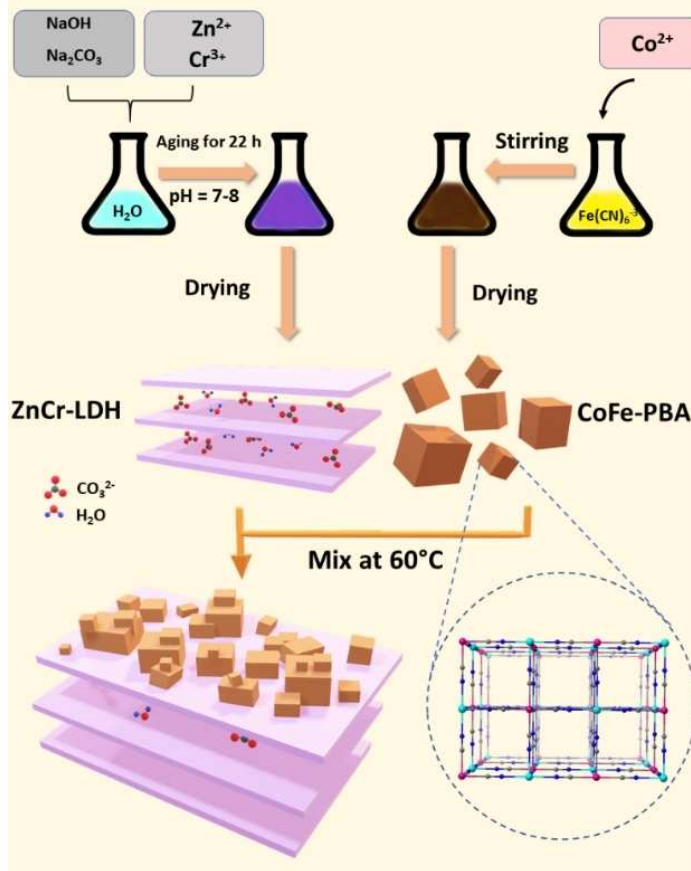

(b)
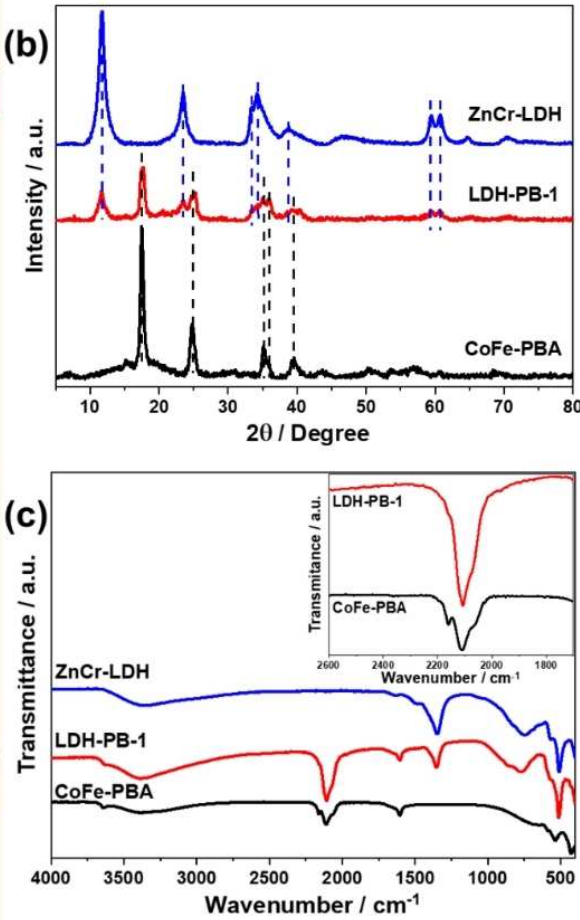

Figure 1. (a) Schematic representation for the preparation of LDH-PB-n hybrid assemblies by mixing $\mathrm{ZnCr}$-LDH and CoFe-PBA at $60^{\circ} \mathrm{C}$ with different weight ratios. (b) XRD patterns of $L D H, P B A$, and LDH-PB-1. The dashed lines represent the characteristic diffraction peaks for $L D H$ and PBA structures in the powder XRD pattern of the LDH-PB-1 sample. (c) ATR-FTIR spectra of LDH, PBA, and LDH-PB-1. Inset: The cyanide stretching mode for CoFe-PBA and LDH-PB-1 samples.

characteristic peaks of a layered double hydroxide and a Prussian blue structure were observed in powder XRD patterns of samples (Figure $1 \mathrm{~b}$ and Figure S1 in the Supporting Information). The diffraction peaks at $11.7^{\circ}$ and $23.5^{\circ}$ that are observed for $\mathrm{ZnCr}-\mathrm{LDH}$ are assigned to (003) and (006) lattice planes of LDH structure while the ones at $17.5^{\circ}$ and $24.8^{\circ}$ observed for CoFe-PBA correspond to (200) and (220) lattice planes of cubic Prussian blue structure, respectively (Table $\mathrm{S} 1) . .^{[17,37]}$ These peaks are also observed for LDH-PB-1 without any significant shift, which suggests a physical interaction between components in LDH-PB-1. The relative intensities of the diffraction peaks that belong to PB structures in LDH-PB-1 decrease and they get broader compared to CoFe-PBA, which indicates that the particle size is reduced during the formation of the hybrid assembly.

The FTIR spectra for $\mathrm{ZnCr}-\mathrm{LDH}$ and LDH-PB- $n$ reveal a broad band at $3370 \mathrm{~cm}^{-1}$ and a sharp one at $1632 \mathrm{~cm}^{-1}$, which are attributed to the $\mathrm{M}-\mathrm{O}-\mathrm{H}$ stretching mode and the bending mode of $\mathrm{H}_{2} \mathrm{O}$. The broadness of the $\mathrm{O}-\mathrm{H}$ band indicates the presence of hydrogen bonds between water molecules and anions in the interlayer space of $\mathrm{LDH}$. The two bands at $1487 \mathrm{~cm}^{-1}$ and $1348 \mathrm{~cm}^{-1}$ are assigned to the asymmetric stretching of the carbonate species placed in between the layers. The spectra for CoFe-PBA and LDH-PB-n reveal a sharp band at $2110 \mathrm{~cm}^{-1}$ with two shoulders at $2160 \mathrm{~cm}^{-1}$ and $2070 \mathrm{~cm}^{-1}$, which are assigned to Co'"-NC-Fe", Co"-NC-Fe"', and $\mathrm{Co} " \mathrm{~N}-\mathrm{NC}-\mathrm{Fe}$ " coordination modes, respectively. The relative decrease in the intensity of the shoulder at $2160 \mathrm{~cm}^{-1}$ for LDH-
PB- $n$ suggests an electron transfer from LDH to PBA structures (Figures $1 \mathrm{C}$ and S2). ${ }^{[38,39]}$

The morphology and structure of the samples were investigated by scanning electron microscopy (SEM) and Transmission electron microscopy (TEM) images. The layered structure of $\mathrm{ZnCr}-\mathrm{LDH}$ was confirmed with SEM analysis (Figure $2 \mathrm{a}){ }_{,}^{[40]}$ which are surrounded by Prussian blue structures after mixing to produce hybrid assemblies (Figure $2 b, c$ ). The morphology of LDH-PB-1 has also been investigated with TEM analysis since it exhibits the highest photocatalytic water oxidation performance (Figure 2d). Scanning TEM (STEM) elemental mapping confirms that CoFe-PBA particles are distributed on the surface of LDH layers in LDH-PB-1 (Figures $2 e$ and S3). ${ }^{[18,41,42]}$ Energy-dispersive $X$-ray spectroscopy (EDS) elemental scanning reveals $\mathrm{Co} / \mathrm{Fe}$ and $\mathrm{Zn} / \mathrm{Cr}$ elemental ratios of $3: 2$ and $2: 1$, respectively, which is consistent with our synthetic procedure (Figure S4 and Table S2). EDS mapping indicates that the PB and LDH structures are well-dispersed to form a uniform hybrid assembly (Figure S5).

The possible electron transfer between LDH and PBA structures is also observed with $\mathrm{X}$-ray photoelectron spectroscopy studies. The corresponding binding energies for $\mathrm{Zn} 2 \mathrm{p}, \mathrm{Cr}$ $2 p$, Co $2 p$, Fe 2p, N 1s, C 1s, and O 1s (Figures S6 and S7) are in good accordance with the expected profiles of CoFe-PBA and $\mathrm{ZnCr}-\mathrm{LDH}$. A comparison of XPS profiles before and after mixing indicates that the binding energies of $\mathrm{Zn} 2 p$ and $\mathrm{Cr} 2 p$ exhibit slightly positive shifts after mixing. ${ }^{[20]}$ Cobalt retains its +2 and +3 oxidation states, ${ }^{[35,43,44]}$ whereas the amount of $\mathrm{Fe}^{2+}$ ions in 

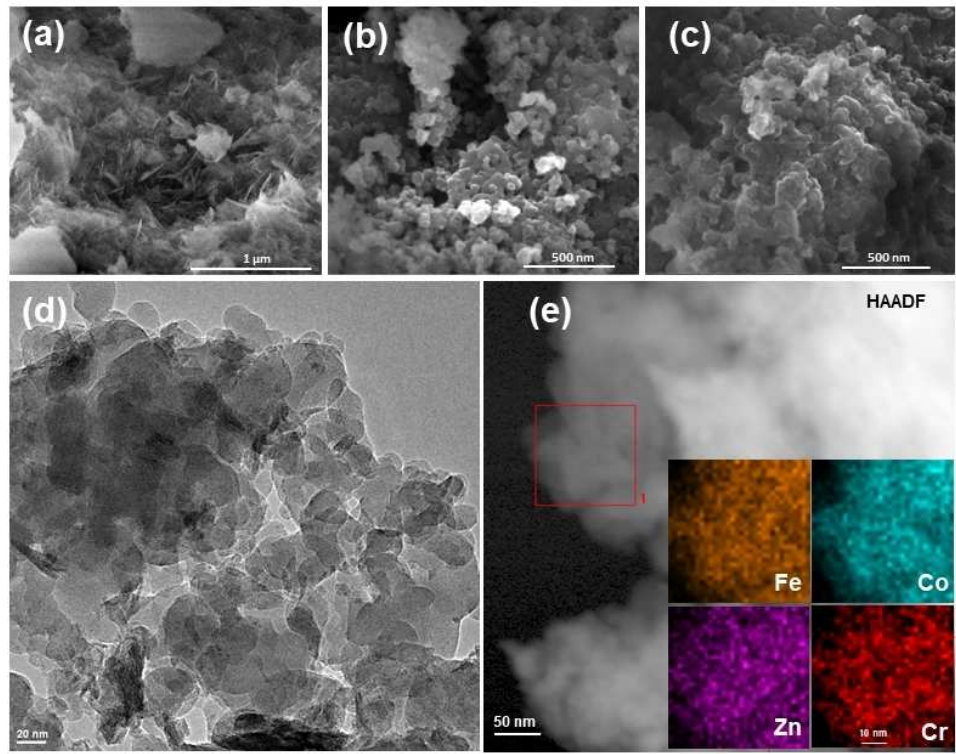

Figure 2. SEM images of (a) ZnCr-LDH, (b) CoFe-PBA, and (c) LDH-PB-1. (d) TEM image of LDH-PB-1. (e) High-angle annular dark field (HAADF) image of the LDH-PB-1 sample. Inset shows the selected-area STEM elemental mapping.

the mixed-valent iron sites increases upon mixing suggesting an electron transfer from LDH to PBA structures (Figures 3 and S8), ${ }^{[27]}$ which is in agreement with FTIR profile of LDH-PB-1 sample.

The photocatalytic performances of bare LDH and LDH-PB- $n$ samples were evaluated by monitoring the amount of gen- erated oxygen from water in the presence of $5 \mathrm{mM} \mathrm{Na}_{2} \mathrm{~S}_{2} \mathrm{O}_{8}$ as a sacrificial agent under visible-light irradiation (Figures $4 \mathrm{a}$ and S9). ZnCr-LDH exhibits a considerable photocatalytic activity, which decreases gradually in the second cycle $\left(0.64 \mathrm{mmol} \mathrm{g}^{-1} \mathrm{~h}^{-1}\right)$. LDH-PB-1 exhibits a threefold increase in the activity $\left(1.83 \mathrm{mmol} \mathrm{g}^{-1} \mathrm{~h}^{-1}\right)$ compared to bare $\mathrm{ZnCr}-\mathrm{LDH}$ and
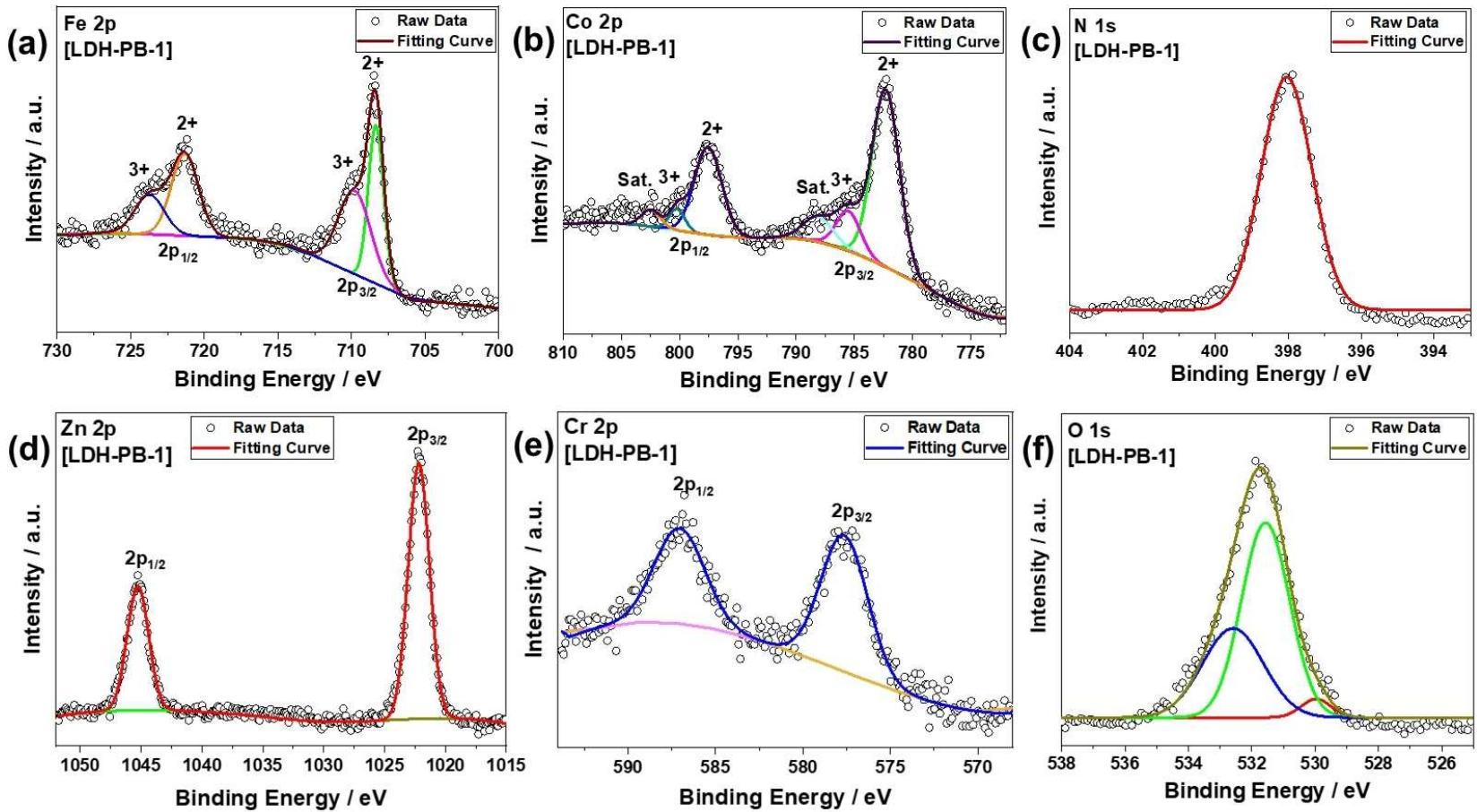

Figure 3. XPS spectra of the LDH-PB-1 sample: (a) Fe 2p; (b) Co 2p; (c) N 1s; (d) Zn 2p; (e) Cr 2p; (f) O 1s. Circles and lines represent raw data and fitted curves, respectively. 

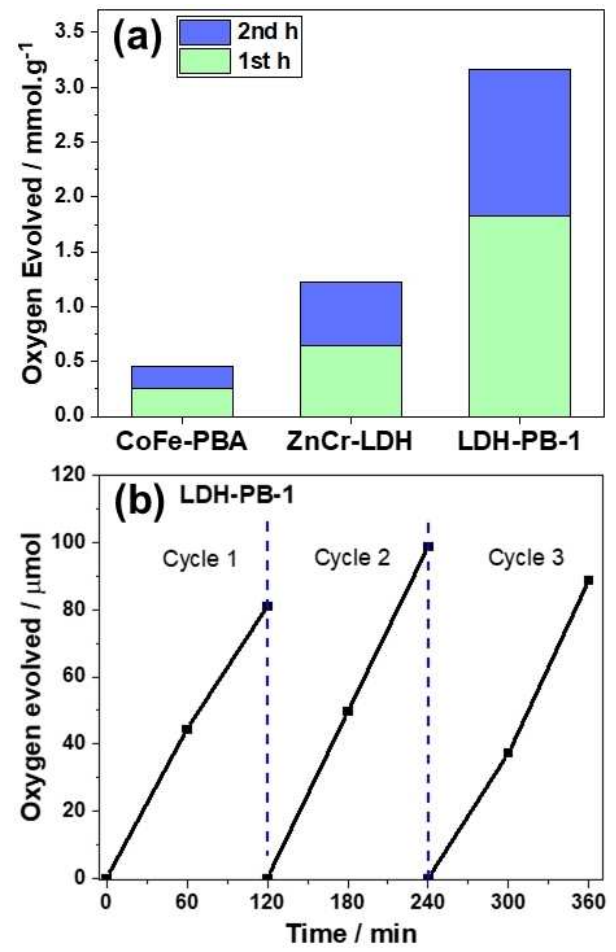

Figure 4. (a) Bar diagrams of photocatalytic $\mathrm{O}_{2}$ evolution of CoFe-PBA, $\mathrm{ZnCr}$ LDH, and LDH-PB-1 (mmol of produced $\mathrm{O}_{2}$ per $\mathrm{g}$ of catalyst) in a pH $7 \mathrm{KPi}$ buffer containing $5 \mathrm{mM} \mathrm{Na}{ }_{2} \mathrm{~S}_{2} \mathrm{O}_{8}$ as a sacrificial agent under visible-light irradiation $\left(\lambda>420 \mathrm{~nm}\right.$ ). (b) Rate of $\mathrm{O}_{2}$ generation in 3 consecutive cycles for LDH-PB-1. After each cycle $5 \mathrm{mM} \mathrm{Na}_{2} \mathrm{~S}_{2} \mathrm{O}_{8}$ was added to the reaction solution.

CoFe-PBA, which maintains its activity even during the third cycle (Figure $4 b)$. The trend in the photocatalytic activity clearly shows that coupling a PBA structure as a WOC not only enhances the activity but also the stability of LDH structures under photocatalytic conditions. The highest photocatalytic activity and stability were obtained when the LDH/PBA molar ratio was 1:1 (LDH-PB-1), which maintains its activity during a $6 \mathrm{~h}$ photocatalytic experiment. When the molar amount of the LDH component is twice that of the PBA one (LDH-PB-2), the activity decreases to approximately half its value $\left(0.64 \mathrm{mmol} \mathrm{g}^{-1} \mathrm{~h}^{-1}\right)$, which could be attributed to the decrease in the weight ratio of the catalyst in the assembly. For LDH-PB3 , which has a molar ratio of $2: 3$, the activity is ca. $0.87 \mathrm{mmol} \mathrm{g}^{-1} \mathrm{~h}^{-1}$ in the first cycle. The activity of LDH-PB-3 in the second cycle is, however, poorer $\left(0.63 \mathrm{mmol} \mathrm{g}^{-1} \mathrm{~h}^{-1}\right)$ due to a possible photodecomposition. The lower stability in LDH-PB-3 could be attributed to a higher degree of aggregation of excess PB particles, which leads to a relatively inefficient electron transfer between components. Furthermore, the physically mixed LDH + PBA sample displays a similar activity to LDH-PB-1 over the first cycle, which decreases significantly in the second cycle (Figure S10) due to the decomposition of the LDH structure as evidenced by post-catalytic XRD studies (Figure S11). These studies indicate that both the synthetic method and the molar ratio of the components play an important role in tuning the physical interaction between the components and, thus, in the activity and photostability. We also studied the effect of the sacrificial agent by evaluating the photocatalytic activity of $\mathrm{ZnCr}$-LDH and LDH-PB-1 with $\mathrm{AgNO}_{3}$ as well. Whereas $\mathrm{ZnCr}$-LDH exhibits a similar activity in the presence of different sacrificial agents, the activity of LDH-PB-1 depends highly on the type of electron scavenger. The activity of LDH-PB-1 is around two times higher in $\mathrm{S}_{2} \mathrm{O}_{8}{ }^{2-}$ compared to $\mathrm{AgNO}_{3}$ since persulfate anion produces an intermediate sulfate radical $\left(\mathrm{SO}_{4}{ }^{-}\right)$, which is a strong oxidizing agent (Figure $\left.\mathrm{S} 12\right) .{ }^{[45]}$

Turnover frequency (TOF) is also an important indicator to compare the activity of the catalytic sites, which is defined as the number of $\mathrm{O}_{2}$ or $\mathrm{H}_{2}$ per catalytic active site per time. ${ }^{[46]}$ For the estimation of the lower-bound TOF value, a rough molecular formula was extracted from EDX analysis and all the cobalt sites are assumed to be catalytically active. The TOF for LDH-PB-1 is obtained to be around $2.1 \times 10^{-4} \mathrm{~s}^{-1}$, which is comparable to previously reported TOF values for Prussian blue-based systems. ${ }^{[27,33,34]}$

The band structure and optical properties of $\mathrm{ZnCr}-\mathrm{LDH}$, CoFe-PBA, and mixed samples were investigated with UV/Vis diffuse-reflectance spectroscopy (Figures $5 a$ and S13). ZnCr-LDH exhibits two strong bands at 410 and $570 \mathrm{~nm}$ corresponding to the ${ }^{4} \mathrm{~A}_{2 g} \rightarrow{ }^{4} \mathrm{~T}_{1 \mathrm{~g}}(\mathrm{~F})$ and ${ }^{4} \mathrm{~A}_{2 \mathrm{~g}} \rightarrow{ }^{4} \mathrm{~T}_{2 \mathrm{~g}}(\mathrm{~F}) \mathrm{d}$-d transitions of chromium ions, respectively. ${ }^{[47,48]}$ The absorption band initiated at $344 \mathrm{~nm}$ can be assigned to the ligand to metal charge transfer (LMCT) from the $\mathrm{O} 2 \mathrm{p}$ orbital to the $\mathrm{Cr} 3 \mathrm{~d}\left(\mathrm{e}_{\mathrm{g}}\right)$ and $\mathrm{Zn} 4 \mathrm{~s}$ orbitals. ${ }^{[49]}$ When it is coupled with CoFe-PBA, the background absorption of the compound was significantly increased due to the electronic interaction between LDH and PBA structures. Two broad absorption regions in CoFe-PBA can be attributed to the charge transfer in the structure. ${ }^{[50]}$ The enhancement in the photocatalytic activity of the LDH-PB-1 sample is also investigated by photoluminescence spectroscopy (PL). In Figure 5b, $\mathrm{ZnCr}$-LDH displays a remarkable depression of $\mathrm{PL}$ peaks upon hybridization with the PBA structure. Taking into account the higher visible light absorption of the LDH-PB-1 sample compared to pristine $\mathrm{LDH}$, the $\mathrm{PL}$ results suggest an efficient electron transfer between LDH and PBA components, which could sufficiently suppress the recombination of photo-generated electrons and holes. ${ }^{[14,51]}$

Cyclic voltammetry (CV) measurements and DRS results were evaluated to estimate the band energy diagram. The literature is contradictory on the assignment of band energy levels of $\mathrm{ZnCr}-\mathrm{LDH}$ mainly due to the presence of $3 \mathrm{~d}^{3}$ states of $\mathrm{Cr}^{3+}$ ions in addition to conduction and valence bands (Table S4). Cyclic voltammetry of $\mathrm{ZnCr}-\mathrm{LDH}$ is utilized to assign the position for the ${ }^{4} \mathrm{~A}_{2 g}$ state at $1.92 \mathrm{~V}$ vs. $\mathrm{NHE}$, whereas the HOMO level of CoFe-PBA is positioned at $1.48 \mathrm{~V}$ vs. NHE (Figure S14). The bandgap of LDH is found to be $3.97 \mathrm{eV}$ based on the fitting of absorption data with the Tauc plot (Figure S15). The HOMO level of PBA is located at a higher energy level compared to the $d\left(t_{2 g}\right)$ level of LDH (Figure $5 c$ ). The electrons in $d\left(t_{2 g}\right)$ orbitals of $L D H$ are excited to the unoccupied interband states by absorbing visible light. As a result, the photogenerated holes can be injected into the HOMO level of PBA, which causes an efficient charge separation. 
(a)

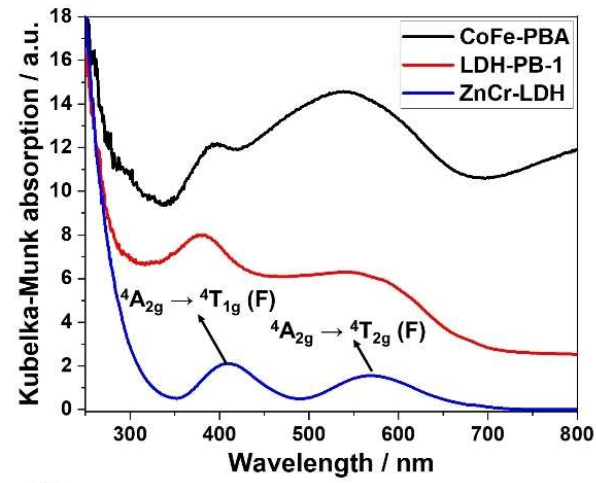

(b)
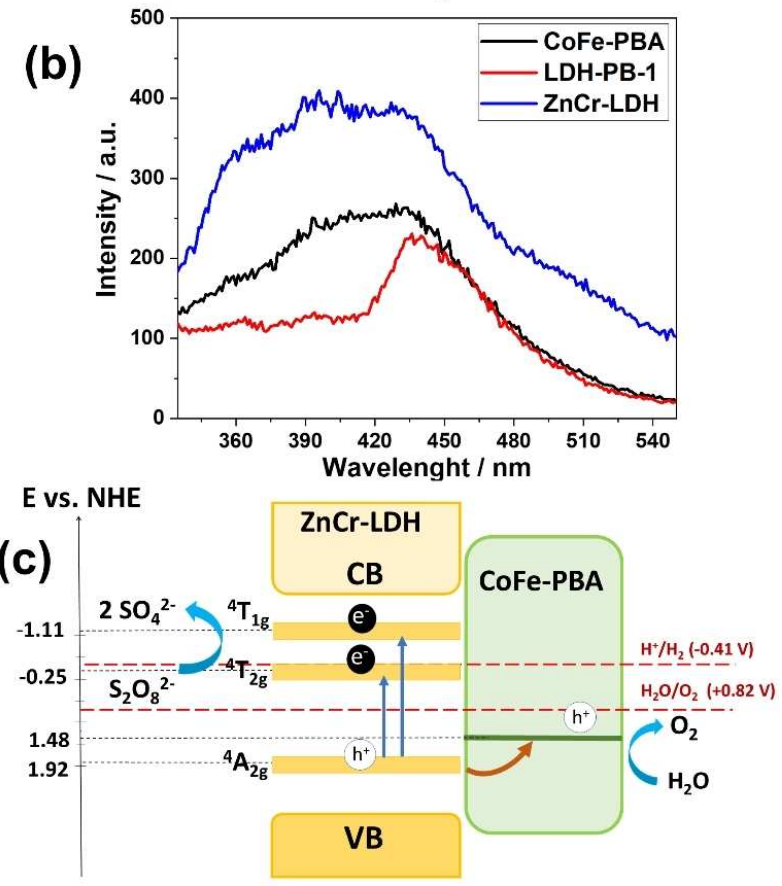

Figure 5. (a) Diffuse-reflectance UV/Vis spectra (plotted as the Kubelka-Munk function of the reflectance, R) of CoFe-PBA, ZnCr-LDH, and LDH-PB-1 samples in the 250-800 nm wavelength range. (b) PL spectra of CoFe-PBA, ZnCr-LDH, and LDH-PB-1 samples $\left(\lambda_{\text {ex }}=290 \mathrm{~nm}\right.$ ) at room temperature. (c) Schematic representation of the estimated band structure of the LDH-PB-1 sample $(\mathrm{pH} 7)$ involving the electron-transfer mechanism.

The robustness of the hybrid assembly is elucidated with XPS and XRD studies performed on post-catalytic powder samples. For the $\mathrm{Cr} 2 \mathrm{p}$ and $\mathrm{Zn} 2 \mathrm{p}$ peaks, a slight shift to higher binding energy was observed. The corresponding $\mathrm{Fe}^{3+}$ peaks are diminished significantly, whereas those for $\mathrm{Fe}^{2+}$ remain unchanged. These results support the thesis that the electron transfer takes place between LDH and PBA during the photocatalytic water oxidation process, which reduces $\mathrm{Fe}^{3+}$ to $\mathrm{Fe}^{2+}$. No significant change in the Co $2 p$ profile is observed in pristine and post-catalytic samples indicating the stability of the $\mathrm{Co}^{2+}$ sites (Figure 6a-d).

XRD patterns were also studied for pristine and postcatalytic powder samples of CoFe-PBA, ZnCr-LDH, and LDH-PB1. Similar to previous studies, the CoFe-PBA sample was observed to be stable during the photocatalytic water oxidation process based on the comparison of XRD profiles of pristine and post-catalytic CoFe-PBA samples (Figure 6e). ZnCr-LDH, however, exhibits additional peaks in the post-catalytic sample, which reveals that it decomposes partially to $\mathrm{K}_{2} \mathrm{Zn}_{4} \mathrm{O}$ $\left(\mathrm{CrO}_{4}\right)_{4} \cdot 3 \mathrm{H}_{2} \mathrm{O}$ under photocatalytic conditions (Figure 6f). The decrease in the activity of $\mathrm{ZnCr}-\mathrm{LDH}$ in the $2 \mathrm{nd}$ cycle could thus be attributed to its low stability. For LDH-PB-1, all the peaks obtained in the pristine and post-catalytic samples correspond to those for PB cubic and LDH structures (Figure $6 \mathrm{~g}$ ). It should be noted that a possible decomposition to an amorphous structure cannot be ruled out merely based on XRD studies. The steady profile of the photocatalytic activity throughout several cycles with LDH-PB-1 and XRD studies performed on pristine and post-catalytic CoFe-PBA, $\mathrm{ZnCr}$-LDH, and LDH-PB-1 samples reveal that the $\mathrm{LDH}$ structure exhibits a remarkable enhancement in the stability once it is coupled with CoFe-PBA to form LDH-PB-1. No additional peaks that can be attributed to a decomposed product are observed in the Infrared spectra of pristine and post-catalytic samples for $\mathrm{ZnCr}$-LDH and LDH-PB-1 (Figure S16). The FTIR spectra of LDH-PB-1 sample before and after the photocatalytic experiment show an electron transfer process between LDH and PBA, which is consistent with XPS studies.

\section{Conclusions}

In summary, the strengths of layered double hydroxides and Prussian blue structures were combined to develop an entirely earth-abundant semiconductor-catalyst assembly for the visible light-driven water oxidation process. We changed experimental parameters including the mixing temperature and weight ratio of components systematically and optimized the synthetic methodology to obtain a combination of enhanced activity and stability. The summary of photocatalytic performances of previously reported $\mathrm{ZnCr}$-LDHs (listed in Table S5) indicates that the LDH-PB assembly exhibits a remarkable activity among LDH-based systems. $\mathrm{ZnCr}$-LDH serves as an efficient visible light-absorbing semiconductor, whereas CoFe-PBA plays an active role in the catalytic water oxidation process. The photocatalytic assembly is easy to prepare and is composed of earthabundant components. The well-placed energy level of CoFe Prussian blue structure between the water oxidation and the valence band of $\mathrm{ZnCr}$-LDH makes it an ideal catalyst for efficient hole transfer from the semiconductor to the water oxidation process. This efficient electron transfer not only reveals a threefold enhancement in photocatalytic performance compared to bare LDH but also improves its stability under photocatalytic conditions. We also observed that the molar ratio of $\mathrm{LDH}$ and PB components can be tuned to enhance the activity and stability of LDH-PB assemblies. Given the remarkable photocatalytic performance of this hybrid assembly and the diversity in both the layered double hydroxide and Prussian blue chemistry, a systematic study on the overall water splitting with LDH-PB assemblies is currently underway. 

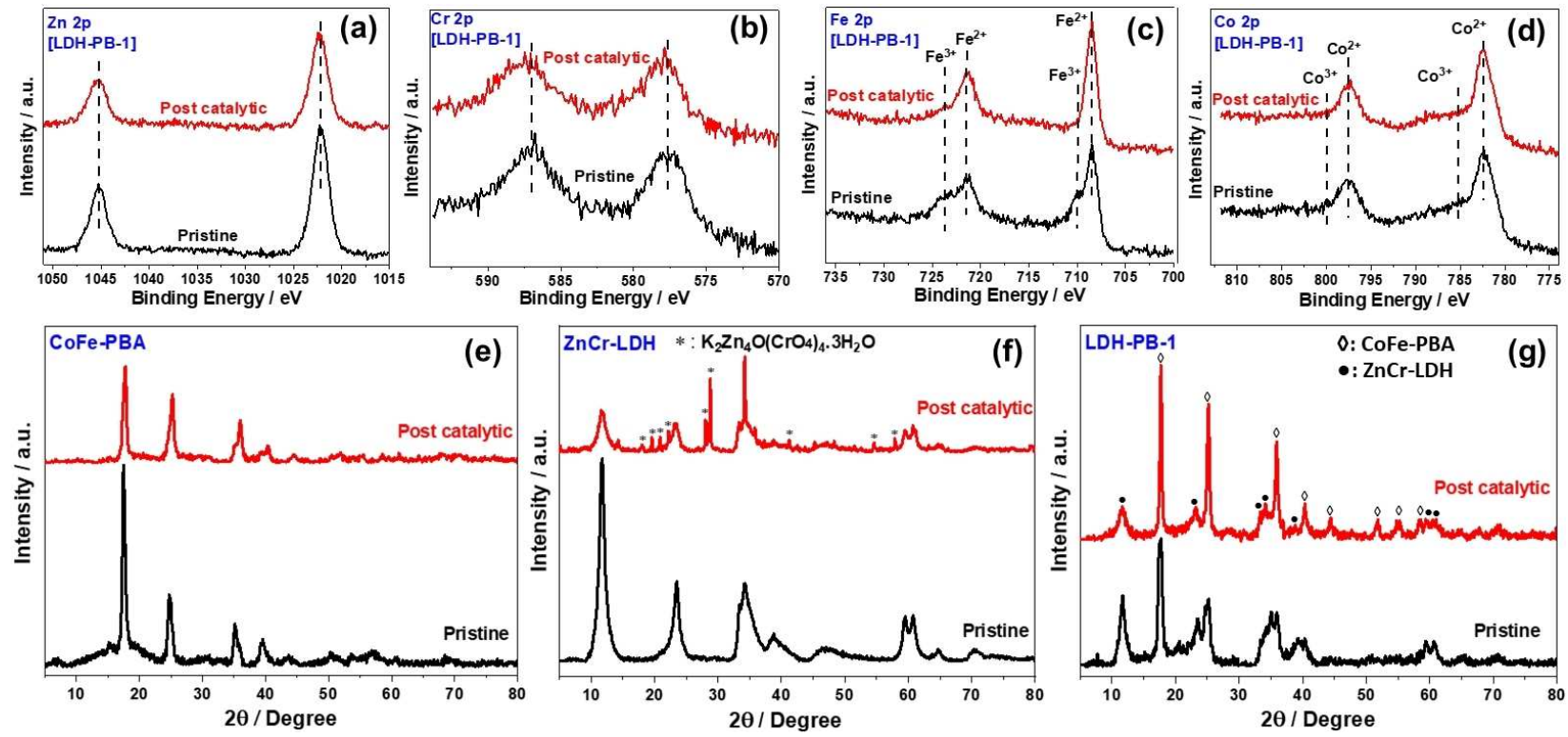

Figure 6. XPS spectra of (a) Zn 2p, (b) $\mathrm{Cr} 2 \mathrm{p}$, (c) Fe 2p, and (d) Co $2 p$ for pristine (black line) and post-catalytic (red line) of LDH-PB-1 sample. XRD patterns of (e) CoFe-PBA, (f) ZnCr-LDH, and (g) LDH-PB-1 for pristine (black line) and post-catalytic (red line) samples.

\section{Experimental Section}

\section{Synthesis of $\mathrm{ZnCr}-\mathrm{LDH}$}

The co-precipitation method was used for the synthesis of $\mathrm{ZnCr}$ $\mathrm{LDH}$, similar to the reported procedure with slight modifications. ${ }^{[18]}$ $\mathrm{Zn}\left(\mathrm{NO}_{3}\right)_{2} \cdot 6 \mathrm{H}_{2} \mathrm{O}$ and $\mathrm{Cr}\left(\mathrm{NO}_{3}\right)_{3} \cdot 6 \mathrm{H}_{2} \mathrm{O}$ with a $\mathrm{Zn} / \mathrm{Cr}$ molar ratio of $2: 1$ were dissolved in deionized water $(30 \mathrm{~mL}$; solution A). Then, solution $\mathrm{A}$ was titrated with another solution containing $1 \mathrm{M} \mathrm{NaOH}$ and $1 \mathrm{M} \mathrm{Na}_{2} \mathrm{CO}_{3}$ (solution $\mathrm{B}$ ) until $\mathrm{pH}$ 7-8 was reached under vigorous stirring at room temperature. The resultant suspension was stirred for $4 \mathrm{~h}$. Subsequently, the mixture was aged at $85^{\circ} \mathrm{C}$ for $22 \mathrm{~h}$. The purple slurry was separated by centrifugation, rinsed with deionized water, and finally dried at $75^{\circ} \mathrm{C}$

\section{Synthesis of CoFe-PBA}

CoFe-PBA was synthesized by a precipitation method. ${ }^{[37]}$ First, $\mathrm{K}_{3}\left[\mathrm{Fe}(\mathrm{CN})_{6}\right](2 \mathrm{mmol})$ was dissolved deionized water $(20 \mathrm{~mL})$. Then, an aqueous solution of $\mathrm{Co}\left(\mathrm{NO}_{3}\right)_{2} \cdot 3 \mathrm{H}_{2} \mathrm{O}(3 \mathrm{mmol}$ in $20 \mathrm{~mL}$ water) was added dropwise to the above solution at room temperature. The obtained slurry was stirred for $1 \mathrm{~h}$ and left to stand overnight. The final precipitate was collected by centrifugation and washed thoroughly with water. The obtained solid was dried at $75^{\circ} \mathrm{C}$.

\section{Synthesis of LDH-PBA mixture}

For the preparation of LDH-PBA assemblies, LDH and PBA powder samples with specific quantities were separately dispersed in a water/ethanol $(1: 1 \mathrm{v} / \mathrm{v})$ mixture and sonicated for $30 \mathrm{~min}$. Then, solutions were mixed with ultrasonication for $2 \mathrm{~h}$. Subsequently, the mixture was stirred at $60^{\circ} \mathrm{C}$ for $6 \mathrm{~h}$ (Figure 1a). The resulting materials with $1: 1,2: 1$, and $2: 3$ weight ratios are denoted as $L D H$ PB-1, LDH-PB-2, and LDH-PB-3, respectively.

\section{Photocatalytic Experiments}

The photocatalytic experiments for oxygen evolution were performed in a $21 \mathrm{~mL}$ Pyrex reactor. Catalysts $(28 \mathrm{mg} ; 14 \mathrm{mg}$ for experiments involving bare PBA and $\mathrm{LDH}$ samples) were dispersed in aqueous buffer solution ( $18 \mathrm{~mL} ; 0.1 \mathrm{M} \mathrm{KH}_{2} \mathrm{PO}_{4}$ and $0.1 \mathrm{M} \mathrm{K}_{2} \mathrm{HPO}_{4}$ diluted in $500 \mathrm{~mL}$ deionized water with a $\mathrm{pH}$ close to 7) containing $5 \mathrm{mM} \mathrm{Na}_{2} \mathrm{~S}_{2} \mathrm{O}_{8}$ as a sacrificial agent. The headspace of the flask was sealed with septa and the reaction mixtures were purged thoroughly with $\mathrm{N}_{2}$ to remove air. A Xe lamp ( 300 W; AM 1.5 global filter.) with a UV cutoff filter $(\lambda>420 \mathrm{~nm})$ was used as a light source. The amount of evolved oxygen was determined by injecting $100 \mu \mathrm{L}$ of the reactor headspace gas into a gas chromatograph (Agilent 7820 A GC) equipped with a Molesieve column and a TCD detector. Ar was used as the carrier gas. For each sample, photocatalytic tests were repeated 3 to 5 times to achieve reliable results.

\section{Acknowledgements}

S.S.A. thanks TUBITAK for support (Project No: 118Z277). F.K. thanks TÜBA-GEBIP for young investigator awards and BAGEP for young scientist awards.

\section{Conflict of Interest}

The authors declare no conflict of interest.

Keywords: layered double hydroxides • photocatalysis Prussian blue - water oxidation - water splitting. 
[1] S. J. A. Moniz, S. A. Shevlin, D. J. Martin, Z. X. Guo, J. Tang, Energy Environ. Sci. 2015, 8, 731-759.

[2] T. Hisatomi, J. Kubota, K. Domen, Chem. Soc. Rev. 2014, 43, 7520-7535.

[3] S. Berardi, S. Drouet, L. Francàs, C. Gimbert-Suriñach, M. Guttentag, C. Richmond, T. Stoll, A. Llobet, Chem. Soc. Rev. 2014, 43, 7501-7519.

[4] T. Takata, K. Domen, ACS Energy Lett. 2019, 4, 542-549.

[5] K. E. Dalle, J. Warnan, J. J. Leung, B. Reuillard, I. S. Karmel, E. Reisner, Chem. Rev. 2019, 119, 2752-2875.

[6] J. W. L. Yeob Lee, J. K. K. Yeob Lee, Jung Hoon Choi, Hyung Joon Jeon, Kyung Min Choi, Energy Environ. Sci. 2011, 4, 914-920.

[7] D. S. Su, M. Wei, X. Duan, B. Wang, Y. Zhao, X. Cao, S. Zhang, P. Chen, L. Tian, D. G. Evans, J. Lu, Z. Li, B. Zhang, Chem. Eur. J. 2012, 18, 11949 11958.

[8] Y. Zhao, B. Li, Q. Wang, W. Gao, C. J. Wang, M. Wei, D. G. Evans, X. Duan, D. O'Hare, Chem. Sci. 2014, 5, 951-958.

[9] H. Zhou, Y. Song, Y. Liu, H. Li, W. Li, Z. Chang, Int. J. Hydrogen Energy 2018, 43, 14328-14336.

[10] S. Nayak, K. M. Parida, Int. J. Hydrogen Energy 2016, 41, 21166-21180.

[11] A. R. Amani-Ghadim, F. Khodam, M. S. Seyed Dorraji, J. Mater. Chem. A 2019, 7, 11408-11422.

[12] Y. Dou, S. Zhang, T. Pan, S. Xu, A. Zhou, M. Pu, H. Yan, J. Han, M. Wei, D. G. Evans, X. Duan, Adv. Funct. Mater. 2015, 25, 2243-2249.

[13] S. M. Xu, H. Yan, M. Wei, J. Phys. Chem. C 2017, 121, 2683-2695.

[14] J. L. Gunjakar, T. W. Kim, H. N. Kim, I. Y. Kim, S. J. Hwang, J. Am. Chem. Soc. 2011, 133, 14998-15007.

[15] C. G. Silva, Y. Bouizi, V. Fornés, H. García, J. Am. Chem. Soc. 2009, 131, 13833-13839.

[16] K. Parida, L. Mohapatra, Dalton Trans. 2012, 41, 1173-1178.

[17] Y. Fu, F. Ning, S. Xu, H. An, M. Shao, M. Wei, J. Mater. Chem. A 2016, 4, 3907-3913.

[18] D. P. Sahoo, S. Nayak, K. H. Reddy, S. Martha, K. Parida, Inorg. Chem 2018, 57, 3840-3854.

[19] C. Wang, B. Ma, S. Xu, D. Li, S. He, Y. Zhao, J. Han, M. Wei, D. G. Evans, X. Duan, Nano Energy 2017, 32, 463-469.

[20] D. P. Sahoo, S. Patnaik, K. Parida, ACS Omega 2019, 4, 14721-14741.

[21] J. L. Gunjakar, I. Y. Kim, J. M. Lee, N. S. Lee, S. J. Hwang, Energy Environ. Sci. 2013, 6, 1008-1017.

[22] J. L. Gunjakar, T. W. Kim, I. Y. Kim, J. M. Lee, S. J. Hwang, Sci. Rep. 2013, 3 2080.

[23] G. Zhang, B. Lin, Y. Qiu, L. He, Y. Chen, B. Gao, Int. J. Hydrogen Energy 2015, 40, 4758-4765.

[24] M. Sohail, H. Kim, T. W. Kim, Sci. Rep. 2019, 9, 7584.

[25] Y. Yamada, K. Oyama, R. Gates, S. Fukuzumi, Angew. Chem. Int. Ed. 2015, 54, 5613-5617; Angew. Chem. 2015, 127, 5705-5709.

[26] F. S. Hegner, I. Herraiz-Cardona, D. Cardenas-Morcoso, N. López, J. R. Galán-Mascarós, S. Gimenez, ACS Appl. Mater. Interfaces 2017, 9, 3767137681.

[27] S. Goberna-Ferrón, W. Y. Hernández, B. Rodríguez-García, J. R. GalánMascarós, ACS Catal. 2014, 4, 1637-1641.

[28] Y. Aratani, K. Oyama, T. Suenobu, Y. Yamada, S. Fukuzumi, Inorg. Chem. 2016, 55, 5780-5786.

[29] L. Han, P. Tang, Á. Reyes-Carmona, B. Rodríguez-García, M. Torréns, J. R. Morante, J. Arbiol, J. R. Galan-Mascaros, J. Am. Chem. Soc. 2016, 138 16037-16045.
[30] B. M. Pires, P. L. Dos Santos, V. Katic, S. Strohauer, R. Landers, A. L. B. Formiga, J. A. Bonacin, Dalton Trans. 2019, 48, 4811-4822.

[31] S. Pintado, S. Goberna-Ferrón, E. C. Escudero-Adán, J. R. Galán-Mascarós, J. Am. Chem. Soc. 2013, 135, 13270-13273.

[32] T. G. Ulusoy Ghobadi, A. Ghobadi, M. C. Soydan, M. Barzgar Vishlaghi, S. Kaya, F. Karadas, E. Ozbay, ChemSusChem 2020, 13, 2577-2588.

[33] Z. Kap, F. Karadas, Faraday Discuss. 2019, 215, 111-122.

[34] T. G. Ulusoy Ghobadi, E. Akhuseyin Yildiz, M. Buyuktemiz, S. Sadigh Akbari, D. Topkaya, Ü. İsci, Y. Dede, H. G. Yaglioglu, F. Karadas, Angew. Chem. Int. Ed. 2018, 17173-17177; Angew. Chem. 2018, 130, 1741917423.

[35] T. G. Ulusoy Ghobadi, A. Ghobadi, M. Buyuktemiz, E. A. Yildiz, D. Berna Yildiz, H. G. Yaglioglu, Y. Dede, E. Ozbay, F. Karadas, Angew. Chem. Int. Ed. 2020, 59, 4082-4090; Angew. Chem. 2020, 132, 4111-4119.

[36] M. Aksoy, S. V. K. Nune, F. Karadas, Inorg. Chem. 2016, 55, 4301-4307.

[37] E. P. Alsaç, E. Ülker, S. V. K. Nune, Y. Dede, F. Karadas, Chem. Eur. J. 2018, 24, 4856-4863.

[38] O. Sato, T. Iyoda, A. Fujishima, K. Hashimoto, Science 1996, 272, 704705.

[39] S. J. Gerber, E. Erasmus, Mater. Chem. Phys. 2018, 203, 73-81.

[40] Y. Xu, L. Tan, Y. Zhao, Y. Song, Ind. Eng. Chem. Res. 2020, 59, 1431514322.

[41] Y. Q. Wang, J. Z. Ma, J. Wang, S. Chen, H. S. Wang, J.T. Zhang, Adv. Energy Mater. 2019, 9, 1802939.

[42] M. N. Shaddad, P. Arunachalam, J. Labis, M. Hezam, A. M. Al-Mayouf, Appl. Catal. B 2019, 244, 863-870.

[43] C. Zhao, B. Liu, X. Li, K. Zhu, R. Hu, Z. Ao, J. Wang, Chem. Commun. 2019 $55,7151-7154$

[44] T. Ivanova, A. Naumkin, A. Sidorov, I. Eremenko, M. Kiskin, J. Electron Spectrosc. Relat. Phenom. 2007, 156-158, 200-203.

[45] A. L. Kaledin, Z. Huang, Y. V. Geletii, T. Lian, C. L. Hill, D. G. Musaev, J. Phys. Chem. A 2010, 114, 73-80.

[46] A. J. Bard, M. A. Fox, Acc. Chem. Res. 1995, 28, 141-145.

[47] J. W. Boclair, P. S. Braterman, J. Jiang, S. Lou, F. Yarberry, Chem. Mater. 1999, 11, 303-307.

[48] J. W. Boclair, P. S. Braterman, Chem. Mater. 1998, 10, 2050-2052.

[49] H. Jiang, K. ichi Katsumata, J. Hong, A. Yamaguchi, K. Nakata, C. Terashima, N. Matsushita, M. Miyauchi, A. Fujishima, Appl. Catal. B 2018, 224, 783-790.

[50] O. Sato, V. Einaga, T. Iyoda, A. Fujishima, K. Hashimoto, J. Electrochem. Soc. 1997, 144, L11.

[51] Z. Y. Yu, Y. Duan, J. D. Liu, Y. Chen, X. K. Liu, W. Liu, T. Ma, Y. Li, X. S. Zheng, T. Yao, M. R. Gao, J. F. Zhu, B. J. Ye, S. H. Yu, Nat. Commun. 2019 10, 2799.

Manuscript received: September 25, 2020

Revised manuscript received: November 4, 2020

Accepted manuscript online: November 6, 2020

Version of record online: November 19, 2020 